\title{
The effects of restricting the flexion-extension motion of the first metatarsophalangeal joint on human walking gait $^{1}$
}

\author{
Junxia Zhang, Ying Si*, Yan Zhang and Yefang Liu \\ School of Mechanical Engineering, Tianjin University of Science and Technology, Tianjin 300222, \\ China
}

\begin{abstract}
The first metatarsophalangeal (MTP) joint is a critical component for normal walking. The present study aimed to investigate the effects of restricting the flexion-extension motion of the first MTP joint on human walking gait. Ten male participants were recruited to perform walking trials under barefoot (BF) and the first MTP joint constraint (FMJC) conditions, respectively. The results showed that there were statistically significant differences in walking speed and stride length. The characteristics of angular displacement in three lower limb joints and ground reaction force (GRF) were almost identical in BF and FMJC. However, maxima, minima and range of motion of three joint angles were significantly different. The utilized coefficient of friction (UCOF) increased significantly after FMJC, indicating that there may be a greater risk of slippage and falling when FMJC.
\end{abstract}

Keywords: The first metatarsophalangeal joint, constraint, gait analysis

\section{Introduction}

In the design of lower extremity exoskeleton, the degrees of freedom of the hip, knee, and ankle are the emphases. The feet of the exoskeleton are usually designed as a whole without the consideration of the flexion-extension motion of the first MTP joint [1,2]. Moreover, in the design of the lower limb prostheses, the motion of this joint is usually limited into a small range. Additionally, in gait analysis, the foot is usually considered as rigid and the flexion-extension of the first MTP joint is ignored. Measurements of the motion of the first MTP joint have been well studied [3-7], but the change of gait parameters related to this joint is less studied.

\footnotetext{
${ }^{1}$ The authors declare that there are no conflicts of interest associated with this work.

*Corresponding author: Ying Si, School of Mechanical Engineering, Tianjin University of Science and Technology, Tianjin 300222, China. Tel.: +86 022-60273203; Fax: +86 022-60273495; E-mail: SiYing163@mail.tust.cn.
}

0959-2989/14/\$27.50 @ 2014 - IOS Press and the authors. 


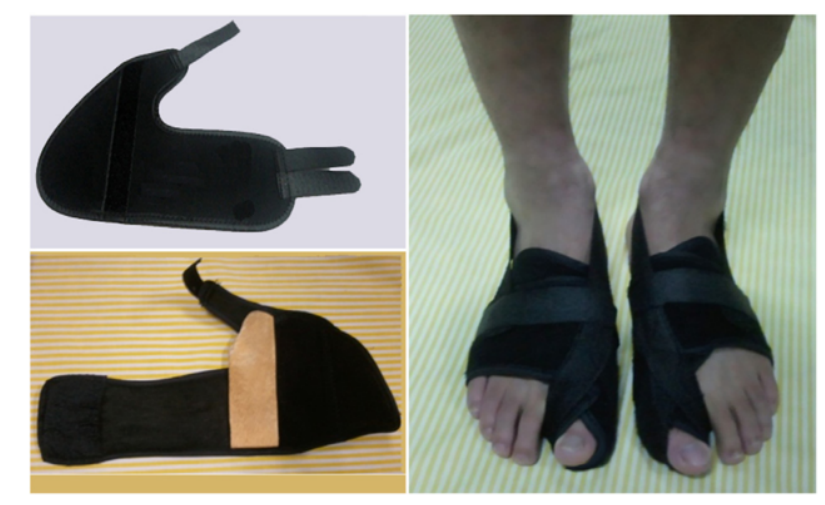

Fig. 1. The restraint device used for the first metatarsophalangeal joint. A small piece of wood was placed beneath the first metatarsophalangeal joint. Also, a big toe extroversion correcting tape was used to fix the position of wood.

The function of the first MTP joint is to maintain the stability of the arch of the foot, bear weight and conduct stress during human walking [8,9]. Adequate motion of the first MTP joint, especially dorsiflexion, is the basic guarantee of an effective pushing of a walk [10]. Moreover, the dorsiflexion of the first MTP joint during gait can be as much as 90 degrees $[3-7,10]$. The loss or restriction of the motion of the first MTP joint can severely impair the function of the foot and change plantar pressure distribution [11]. Studying changes induced by FMJC is critical for understanding the importance of the first MTP joint in human walking.

In this study, the effects of restricting the flexion-extension motion of the first MTP joint on walking gait were investigated. We used a custom fabricated wood splint to restrict the flexion-extension motion of the first MTP joint. And we hypothesized that the gait characteristics would be affected by the restriction. This study will be useful for physical therapy, gait planning for exoskeletons and the design of lower limb prostheses.

\section{Methods}

\subsection{Participants}

We recruited ten healthy young male volunteers (age $25.5 \pm 1.38$ years; height $173.83 \pm 3.92 \mathrm{~cm}$; body mass $65.33 \pm 3.67 \mathrm{~kg}$ ) to perform the test. The institutional review board of the Tianjin University of Science and Technology approved this study and all subjects gave written informed consent. All of the participants have not any neurological or orthopedic conditions, previous back injuries, recent fractures, muscle strains, or joint sprains, and are capable of independent ambulation.

\subsection{Test equipment}

In order to restrict the flexion-extension motion of the first MTP joint, a custom fabricated wood splint (Figure 1) was fabricated based on a type of big toe extroversion correcting tape. A small piece of wood $(10.0 \mathrm{~cm} \times 3.5 \mathrm{~cm} \times 0.3 \mathrm{~cm})$ was placed beneath the first MTP joint in the neutral position. Correcting tape was used to fix the position of the wood. 
The walking trials were performed on a 5-m walkway. Data capturing took place at the middle 2.5 $\mathrm{m}$ of the walkway, thus excluding any accelerations or decelerations. Three-dimensional motion was captured using a six-camera motion analysis system (VICON, Oxford Metrics Ltd., Oxford, UK). Sixteen retro-reflective markers (14 $\mathrm{mm}$ in diameter) were attached to the participant's lower limbs according to the Plug- In-Gait marker set. The motion data were sampled at $120 \mathrm{~Hz}$.

Ground reaction forces (GRF) were recorded using two AMTI force plates (OR6-6-1, AMTI Corp., Newton, MA, USA). The force plate data were sampled at $1200 \mathrm{~Hz}$. The VICON and AMTI were time-synchronized.

\subsection{Protocol}

Before the trials, the age, height, weight, and lower-extremity anthropometric data of the participants were measured. The subjects were permitted multiple practice trials $(5$ trials in average per subject) to acclimate to the environment, the markers, and the restraint device. After the acclimation period, the subjects were required to complete 10 barefoot (BF) trials and 10 first MTP joint constraint (FMJC) trials. The subjects were instructed to perform each trial normally. After every two walking trials, each subject would be asked to rest for 2 min to avoid fatigue effect.

\subsection{Data analysis}

A gait cycle, is divided by the heel contact and toe-off, includes the stance phase and swing phase. Single support phase (one foot stands on the ground) and double support phase (both feet in contact with the ground) are included in stance phase. Walking time was normalized to a gait cycle as follows: $0 \%$ represented the first heel contact with the ground of the leading foot (right foot), and 100\% represented the next heel contact of the leading foot. Data acquired from the markers and force plates were used to calculate spatiotemporal parameters, which included walking speed $(\mathrm{m} / \mathrm{s})$, stride length (m), cadence (steps/min), single support time(s) and double support time(s), and joint angles of lower limbs through the Plug-In-Gait Biomechanical Modeler Pipeline (Oxford Metrics, Oxford, UK) [12]. The GRF were normalized to the subject's body weight. During the GRF analysis, time was normalized to the stance phase as follows: $0 \%$ represented heel contact, and $100 \%$ represented toe off the ground [13]. Motions in sagittal plane, which divides the body into two left and right parts, were analyzed as the majority of the motions occur in this plane [14].

During walking, a slip is likely to occur when the utilized coefficient of friction (UCOF), also referred to as the 'required' coefficient of friction (RCOF), of an individual exceeds the available friction at the foot floor interface [15-17]. Normalized GRF were used to calculate a subject's UCOF. For each trial, the UCOF was calculated by dividing the resultant shear force (computed from the anterior-posterior $\left(\mathrm{F}_{\mathrm{Y}}\right)$ and the medial-lateral $\left(\mathrm{F}_{\mathrm{X}}\right)$ forces) by the vertical force $\left(\mathrm{F}_{\mathrm{Z}}\right)$, as shown in Eq. (1). In order to avoid spurious UCOF values resulting from the division by small numbers, the data were screened, and only the UCOF data in which the vertical GRF exceeded $50 \mathrm{~N}$ were analyzed [18].

$$
\mathrm{UCOF}=\frac{\sqrt{F_{\mathrm{X}}^{2}+F_{Y}^{2}}}{F_{Z}}
$$


Table 1

Spatiotemporal parameters obtained from the BF and FMJC trials

\begin{tabular}{llll}
\hline Spatiotemporal Parameters & BF & FMJC & $P$-value \\
\cline { 2 - 4 } & Mean \pm SD & Mean \pm SD & $0.041^{*}$ \\
Walking Speed (m/s) & $1.20 \pm 0.04$ & $1.16 \pm 0.04$ & $0.009^{*}$ \\
Stride Length (m) & $1.31 \pm 0.02$ & $1.28 \pm 0.03$ & 0.382 \\
Cadence (steps/min) & $109.61 \pm 2.81$ & $108.78 \pm 2.38$ & 0.102 \\
Single Support Time(s) & $0.44 \pm 0.01$ & $0.44 \pm 0.01$ & 0.054 \\
Double Support Time(s) & $0.22 \pm 0.02$ & $0.23 \pm 0.02$ & \\
\hline
\end{tabular}

*indicates significant changes. BF: barefoot walking. FMJC: the first metatarsophalangeal joint constraint walking.

The gait parameters were analyzed using paired $t$-test to verify the significant differences between $\mathrm{BF}$ and FMJC walking. $P$-values $<0.05$ were considered significant. All of the data analyses were conducted using Statistical Product and Service Solution (SPSS) 20.0 statistical software.

\section{Results}

\subsection{Spatiotemporal parameters}

The walking speed $(p=0.041)$ and stride length $(p=0.009)$ decreased significantly after the first MTP joint was restricted (Table 1). The other parameters had no significant changes.

\subsection{Kinematic parameters}

The characteristics of the angular displacement of the ankle, knee, and hip were almost identical in the BF and FMJC trials (Figure 2). However, maxima, minima and range of motion of joint angles were significantly different (Table 2).

The maximum plantar flexion of the ankle joint in loading response $\left(\mathrm{AS}_{1}, p<0.05\right.$; Figure 2a, Table 2) in FMJC was significantly smaller than in BF. The ankle joint was significantly dorsi flexed during stance and swing phases in FMJC $\left(\mathrm{AS}_{2}\right.$ and $\mathrm{AS}_{3}, p<0.05$; Figure 2a, Table 2). The total range of motion of the ankle joint was also significantly different $\left(\mathrm{AS}_{4}, p<0.05\right.$; Table 2$)$.

The maximum flexion of the knee joint significantly increased in loading response and during swing after FMJC $\left(\mathrm{KS}_{1}\right.$ and $\mathrm{KS}_{2}, p<0.05$; Figure $2 \mathrm{~b}$, Table 2$)$. However, the total range of motion of the knee had no significant difference $\left(\mathrm{KS}_{4}, p>0.05\right.$; Table 2$)$.

The hip joint in FMJC exhibited more flexion $\left(\mathrm{HS}_{1}, p<0.05\right.$; Figure $2 \mathrm{c}$, Table 2$)$ at heel strike, but less extension $\left(\mathrm{HS}_{2}, p<0.05\right.$; Figure $2 \mathrm{c}$, Table 2$)$ during stance phase. The total range of motion of the hip in FMJC was greater than in $\mathrm{BF}\left(\mathrm{HS}_{3}, p<0.05\right.$; Table 2$)$.

\subsection{Ground reaction force}

The vertical GRF curves in both the BF and the FMJC trials exhibited two main peaks during stance. Basing on Figure 3, the first peak obtained in the FMJC trials was nearly identical with that obtained in the BF trials, but the second FMJC peak was higher than BF (Figure 3). 


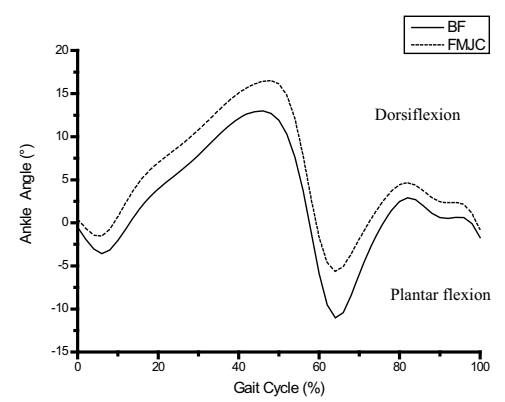

(a) Ankle

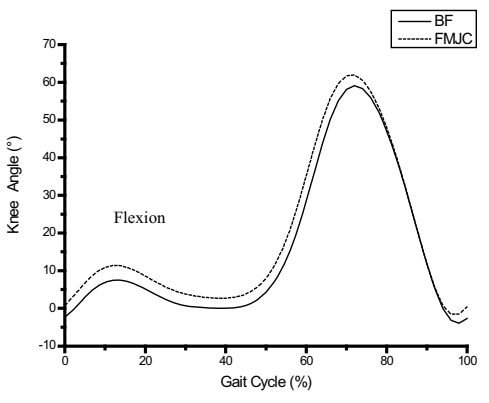

(b) Knee

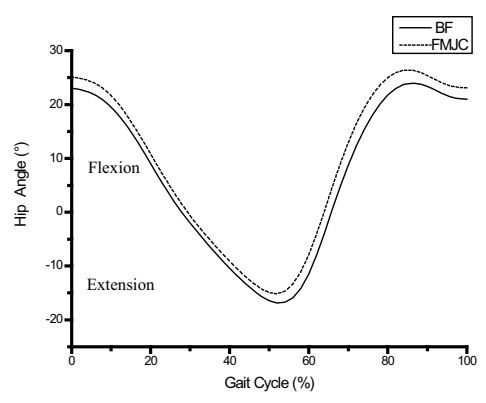

(c) Hip

Fig. 2. Mean joint angles of ankle, knee and hip during BF and FMJC. BF: barefoot walking. FMJC: the first metatarsophalangeal joint constraint walking.

Table 2

Kinematic parameters in the sagittal plane

\begin{tabular}{lllll}
\hline \multirow{2}{*}{ Variable } & Event & BF & FMJC & \\
\cline { 3 - 4 } & & Mean \pm SD & Mean \pm SD & \\
\hline Ankle variable & & & & \\
$\mathrm{AS}_{1}$ & Max. plantar flexion in loading response $\left(^{\circ}\right)$ & $-3.57 \pm 2.38$ & $-1.52 \pm 1.73$ & $<0.001^{*}$ \\
$\mathrm{AS}_{2}$ & Max. dorsiflexion during stance $\left(^{\circ}\right)$ & $12.99 \pm 3.13$ & $16.53 \pm 1.90$ & $<0.001^{*}$ \\
$\mathrm{AS}_{3}$ & Max. plantar flexion during swing $\left(^{\circ}\right)$ & $-11.05 \pm 1.68$ & $-5.65 \pm 2.57$ & $<0.001^{*}$ \\
$\mathrm{AS}_{4}$ & Total range of motion $\left(^{\circ}\right)$ & $24.78 \pm 2.23$ & $23.16 \pm 2.58$ & $0.02^{*}$ \\
$\mathrm{Knee} \mathrm{variable}$ & & & \\
$\mathrm{KS}_{1}$ & Max. flexion in loading response $\left(^{\circ}\right)$ & $7.48 \pm 5.81$ & $11.40 \pm 6.53$ & $<0.001^{*}$ \\
$\mathrm{KS}_{2}$ & Max. flexion during swing $\left(^{\circ}\right)$ & $59.11 \pm 4.58$ & $61.95 \pm 2.09$ & $0.003^{*}$ \\
$\mathrm{KS}_{3}$ & Total range of motion $\left(^{\circ}\right)$ & $65.74 \pm 3.37$ & $65.68 \pm 1.27$ & 0.945 \\
$\mathrm{Hip}$ variable & & & & $0.001^{*}$ \\
$\mathrm{HS}_{1}$ & Flexion at heel strike $\left(^{\circ}\right)$ & $23.06 \pm 3.50$ & $25.12 \pm 5.21$ & $0.001^{*}$ \\
$\mathrm{HS}_{2}$ & Max. extension during stance $\left(^{\circ}\right)$ & $-16.84 \pm 5.17$ & $-15.14 \pm 6.55$ & $0.018^{*}$ \\
$\mathrm{HS}_{3}$ & Total range of motion & $41.72 \pm 3.42$ & $42.73 \pm 3.94$ & \\
\hline
\end{tabular}

*indicates significant changes. BF: barefoot walking. FMJC: the first metatarsophalangeal joint constraint walking.

\section{4. $U C O F$}

A significant increase in the UCOF was observed after FMJC. And the UCOF in the FMJC trials is $23 \%$ greater than in BF (Table 3 ).

\section{Discussion}

Our results showed that the walking speed and stride length of the participants both decreased significantly after the first MTP joint was restricted. Similar results were found by Laroche et al. [19].

They found that the walking velocity was lower and the stride length shorter in rheumatoid arthritis patients than in controls. Besides, previous studies have shown that those two parameters have effects 


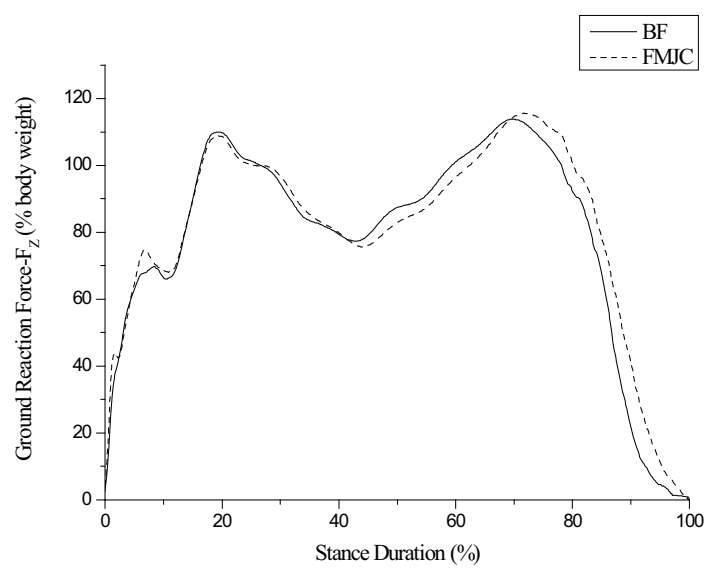

Fig. 3. Mean GRF curves during BF and FMJC. BF: barefoot walking. FMJC: the first metatarsophalangeal joint constraint walking.

Table 3

UCOF measured during the BF and FMJC trials

\begin{tabular}{llll}
\hline \multirow{2}{*}{ UCOF } & BF & FMJC & $\begin{array}{l}\text { Significance } \\
\text { (two-tailed) }\end{array}$ \\
\cline { 2 - 4 } & Mean \pm SD & Mean \pm SD & $0.001^{*}$ \\
\hline Dry level walking & $0.22 \pm 0.05$ & $0.27 \pm 0.05$ & \\
\hline
\end{tabular}

*indicates significant changes. BF: barefoot walking. FMJC: the first metatarsophalangeal joint constraint walking.

on gait performance. Specifically, Chung et al. [20] found that the gait performance while walking generally increased with the increasing walking speed; Kirtley et al. [21] have found that peak knee flexion moment is strongly related to walking speed, and stride length is probably the best basis for deciding the normal range for a particular measurement; Meir Plotnik et al. [22] founded that left-right anti-phase stepping was similar in normal and fast walking, but altered during slow walking. So, it is suggested that restricting the flexion-extension motion of the first MTP joint could change normal gait performance.

The kinematic results showed how the motions of lower limb joints would be modified by FMJC. The higher dorsiflexion of the ankle joint was generated in FMJC during the terminal stance and pre-swing phases of the gait than in BF. Ankle dorsiflexion would reserve and then provide toe-pushing force to help body mass move forward [10,23]. So, greater dorsiflexion may provide stronger pushing forces to perform normal walking. Increased flexion of the knee and the hip were observed in FMJC walking. And those results were consistent with the study of Laroche et al. [19]. Their study showed that there was a negative relationship between maximal flexion of the knee and hips during walking and the underlying MTP dorsal flexion range of motion. The compensatory motion of lower limb joints enabled the participants with FMJC to walk with a walking speed $97 \%$ $(100 *(1.16 / 1.20)$, Table 1$)$ of BF and a stride length $98.0 \%(100 *(1.28 / 1.31)$, Table 1$)$ of BF.

Restricting the flexion-extension motion of the first MTP joint would enlarge the rocker radii of foot. As a result, roll-over shapes may be changed. However, the report of Hansen et al. [24] showed that roll-over shapes in able-bodied subjects do not change appreciably for conditions of level ground walking, when wearing shoes with different rocker radii. Since plantar pressure can decide roll-over 
shapes and vertical GRF, the feature of roll-over shapes mentioned may be the reason why the GRF curves of BF and FMJC showed almost the same characteristic.

In one condition, the available friction at the foot floor interface is a constant; therefore, a greater UCOF reflects a greater risk for slipping and falling [15-17]. The UCOF in FMJC was significantly greater than in $\mathrm{BF}$, indicating that restricting the flexion-extension motion of the first MTP joint can induce greater risk of slippage and falling.

The purpose of this study was to explore the effects of restricting the flexion-extension motion of the first MTP joint on human walking gait. A restriction, not immobilization, was applied in the present study. And the results we got proved the existence of FMJC effects. Next, studying the relationship between the level of restriction and gait performance would be the conducted.

\section{Conclusion}

The aim of this study was to investigate the importance of the flexion-extension motion of the first MTP joint by means of restricting this motion. The results showed that the walking speed and stride length decreased significantly after the first MTP joint was restricted. The characteristics of angular displacement in three lower limb joints and GRF exhibited no significant differences between the BF and the FMJC walking. However, maxima, minima and range of motion of the joint angles, were significantly different. The analysis of the UCOF indicated that FMJC can induce greater risk of slippage and falling. Generally, the flexion-extension motion of the first MTP joint is important for normal walking and should be considered by related researchers.

\section{Acknowledgement}

The authors thank Juan Ge, Wenwu Wang and Shengxian Wang for their assistance in various aspects of the present work. The authors are grateful to all of the subjects who participated in this study. The authors acknowledge the financial support provided by the National Natural Science Foundation of China (50975204).

\section{References}

[1] A.M. Dollar and H. Herr, Lower extremity exoskeletons and active orthoses: challenges and state-of-the-art, IEEE Transactions on Robotics 24 (2008), 144-158.

[2] Ekso, redefining gait training and rehabilitation, available: http://eksobionics.com/, Accessed 15 April 2014, 2010.

[3] T. Buell, D.R. Green and J. Risser, Measurement of the first metatarsophalangeal joint range of motion, J. Am. Podiat. Med. Assn.78 (1988), 439-448.

[4] M.M. Hopson, T.G. McPoil and M.W. Cornwall, Motion of the first metatarsophalangeal joint, Reliability and validity of four measurement techniques, J. Am. Podiat. Med. Assn. 85 (1995), 198-204.

[5] A.D. Milne, D.G. Chess, J.A. Johnson and G.J. King, Accuracy of an electromagnetic tracking device: a study of the optimal range and metal interference, J. Biomech. 29 (1996), 791-793.

[6] J.G. Sammarco, Biomechanics of the foot, in: Basic Biomechanics of the Skeletal System, 2nd ed., M. Nordin and V.H. Frankel, eds., Lea and Febiger, Philadelphia, 1989, pp.170.

[7] M.J. Shereff, F.J. Bejjani and F.J. Kummer, Kinematics of the first metatarsophalangeal joint, J. Bone and Joint Surg. 68 (1986), 392-398.

[8] D.A. Bruening, K.M. Cooney and F.L. Buczek, Analysis of a kinetic multi-segment foot model part II: Kinetics and clinical implications, Gait \& Posture 35 (2012), 535-540. 
[9] A. Boonpratatong and L. Ren, The human ankle-foot complex as a multi-configurable mechanism during the stance phase of walking, J. Bionic Engineering 7 (2010), 211-218.

[10] D.A. Nawoczenski, J.F. Baumhauer and B.R. Umberger, Relationship between clinical measurements and motion of the first metatarsophalangeal joint during gait, J. Bone and Joint Surg. 81-A (1999), 370-376.

[11] H.B. Menz and M.E. Morris, Clinical determinants of plantar forces and pressures during walking in older people, Gait \& Posture 24 (2006), 229-36.

[12] M. Lee, J. Kim, J. Son and Y. Kim, Kinematic and kinetic analysis during forward and backward walking, Gait \& Posture 38 (2013), 674-8.

[13] R. Cham and M.S. Redfern, Changes in gait when anticipating slippery floors, Gait \& Posture 15 (2002): $159-171$.

[14] S.H. Scott and D.A. Winter, Talocrural and talocalcaneal joint kinematics and kinetics during the stance phase of walking, Journal of Biomechanics 24 (1991), 743-752.

[15] J.M. Burnifield and C.M. Powers, Prediction of slips: an evaluation of utilized coefficient of friction and available slip resistance, Ergonomics 49 (2006), 982-995.

[16] J.P. Hanson, M.S. Redfern and M. Mazumdar, Predicting slips and falls considering required and available friction, Ergonomics 42 (1999), 1619-1633.

[17] B.T. Kulakowski, F.L. Buczek, P.R. Cavanagh and P. Pradhan, Evaluation of performance of three slip resistance testers, J. Testing and Evaluation 17 (1989), 234-240.

[18] F.L. Buczek and S.A. Banks, High-resolution force plate analysis of utilized slip resistance in human walking, J. Testing and Evaluation 24 (1996), 353-358.

[19] D. Laroche, T. Pozzo, P. Ornetti, C. Tavernier and J.F. Maillefert, Effects of loss of metatarsophalangeal joint mobility on gait in rheumatoid arthritis patients, Rheumatology 45 (2006), 435-40.

[20] M.J. Chung and M.J. Wang, The change of gait parameters during walking at different percentage of preferred walking speed for healthy adults aged 20-60 years, Gait \& Posture 31 (2010), 131-135.

[21] C. Kirtley, M.W. Whittle and R.J. Jefferson, Influence of walking speed on gait parameters, Journal of Biomedical Engineering 7 (1985), 282-288.

[22] M. Plotnik, R.P. Bartsch, A. Zeev, N. Giladi and J.M. Hausdorff, Effects of walking speed on asymmetry and bilateral coordination of gait, Gait \& Posture 38 (2013), 864-869.

[23] U. Waldecker, Pedographic analysis of hallux valgus deformity, Foot Ankle Surg. 10 (2004), 121-124.

[24] A.H. Hansen and D.S. Childress, Investigations of roll-over shape: implications for design, alignment, and evaluation of ankle-footprostheses and orthoses, Disabil. Rehabil. 32 (2010), 2201-9. 